\title{
Service Level Agreement Characteristics of Monitoring Wireless Sensor Networks for Water Resource Management (SLAs4Water)
}

\author{
George IORDACHE ${ }^{*}$, Adrian PASCHKE ${ }^{2}$, Mariana MOCANU1 ${ }^{1}$, Catalin NEGRU1 \\ ${ }^{1}$ University Politehnica of Bucharest, Computer Science Department, 313 Spl. Independenței, Bucharest- \\ Sector 6, Bucharest, 60042, Romania \\ george.iordache@cs.pub.ro (*Corresponding author),mariana.mocanu@cs.pub.ro, catalin.negru@cs.pub.ro \\ ${ }^{2}$ Fraunhofer FOKUS and Freie Universitaet Berlin, Berlin, Germany \\ paschke@inf.fu-berlin.de
}

\begin{abstract}
One of the most important characteristics of a Service Level Agreement (SLA) when discussing about Monitoring Wireless Sensor Networks (MWSNs) is its effectiveness in assuring business success, a high provider profit, an increased level of client satisfaction and trust. In order to ensure that these goals will be achieved the provider of the MWSN must define some parameters that characterize the Service Level Agreement that occurs between MWSN provider and the MWSN customer. This paper addresses a solution behind an efficient and effective Service Level Agreement (SLA) design and implementation related to a Monitoring Wireless Sensor Network (MSN) and applies the SLA in the case of water resources management. The characteristics of the SLA in place between the MWSN provider and the MWSN customer must be defined by taking into consideration various parameters that are particular to the MWSN such as routing algorithms, recovery from failure, monitoring and reporting aspects. An appropriate definition of a SLA contract helps identify the responsibilities of both the provider and customer of the Monitoring Wireless Sensor Network (MSN) and makes the interaction between the provider and customer easier.
\end{abstract}

Keywords: Service Level Agreement, Service Level Agreement characteristics, Monitoring Wireless Sensor Networks, Water Quality Monitoring.

\section{Introduction}

Water monitoring infrastructures use components such as: supervisory, control and data acquisition systems, wireless sensors or smart meters producing data in different formats and scales. Data can come from geographic information systems, or third-party sources such as water users. So, monitoring of water distribution systems implies large volume of heterogeneous information with temporal dimensions. It is mandatory to transmit and analyses data efficiently to respond in realtime and to alert affected population in case of a pollution accident [13].

A Service Level Agreement (SLA) is a contract between two parts (the provider and the customer) that occurs when the customer requests and receives from a given provider (as a service) an operating Monitoring Wireless Sensor Network (MWSN) to use the results provided by the MWSN with a certain purpose. When discussing about such a contract we refer to different parameters (see Table 1) of the MWSN that can be usually measured. Given these observations we can refer to the SLA as a common contract between the customer and the provider that has the purpose of verifying the functional aspects of the MWSN parameters. The MWSN parameters are measured and monitored to assure certain characteristics of the service such as an increased level of customer satisfaction and Quality of Service, high performance and high availability, as well as cost reduction. This article describes the characteristics of a SLA both in terms of its lifecycle and in terms of parameters that must be specified in such a contract.

For the SLAs in the Web Services domain a specification was given, in XML format, in the WSLA framework [10].

This article is organized as follows: Section 1 presents a short introduction related to water monitoring and SLA. Section 2 sets forth the SLA lifecycle. In Section 3 several SLA parameters related to water monitoring resources are presented. In Section 4 a few use cases related to SLA for water monitoring are presented while Section 5 provides the conclusions of this paper.

\section{Related work}

In [6] the authors give formal guidelines for the implementation of a SLA architecture for operated WSNs. The architecture is designed to distinguish the formal algorithms necessary to operate a WSN according to SLAs. 
In [7] is presented a guideline to write a SLA for Internet of Things operation. According to the authors the volume growth of connected devices opens the door for wireless sensors networks. The authors extend the specification with specific items that integrate the SLA framework with WSN constraints.

In [2] and [3] the authors show SLA-based specifications and usage parameters that are based on the access and configuration of wireless sensor networks. The usage parameters are illustrated both from the point of view of customers and from the point of view of wireless sensor network providers.

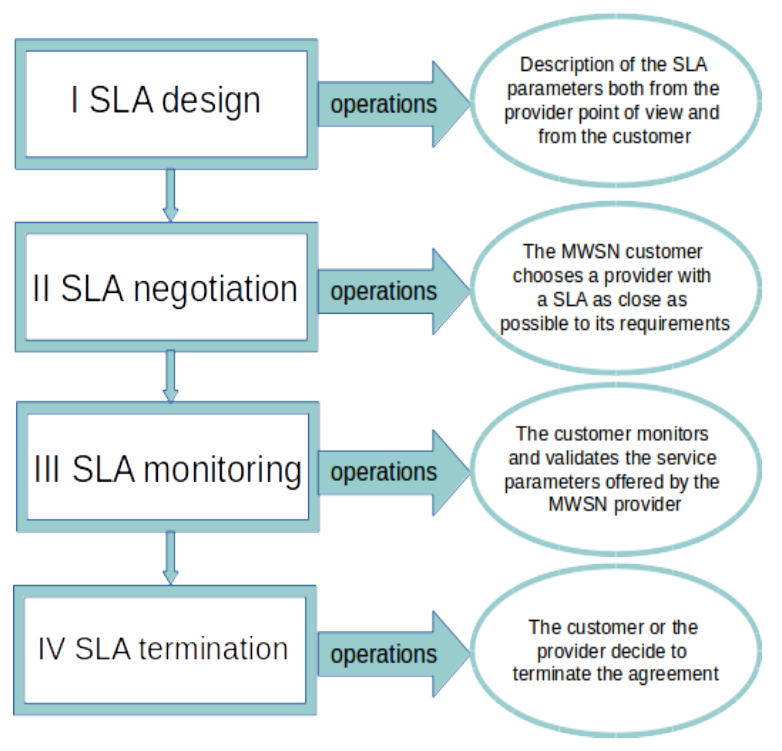

Figure 1. The SLA lifecycle

\section{SLA Lifecycle}

When discussing about a SLA contract we refer to different parameters (see Table 1) of the MWSN that can be usually measured. Given these observations we can refer to the SLA as a common contract between the customer and the provider that has the purpose of verifying the functioning of the MWSN parameters. The MWSN parameters are measured and monitored with the purpose of assuring certain characteristics of the service such as an increased level of customer satisfaction and Quality of Service, high performance and high availability, and cost reduction. This article describes the characteristics of a Service Level Agreement both in terms of its lifecycle and in terms of parameters that must be specified in such a contract. When thinking about the SLA lifecycle we can take into consideration four steps (phases) [9]. We can adapt the four steps from [9] to the case of a SLA contract for a MWSN as follows (see Figure 1). The first step of an SLA is the design of the contract. During this step, on the one hand, the service customers describe the requirements they have in terms of SLA contracts characteristics and on the other hand, several MWSN service providers offer SLA contracts descriptions based on the parameters of their services.

- The next step of the SLA lifecycle is the SLA selection. In this step, the MWSN service customer considers various MWSN services from possible providers and chooses the SLA(s) that is (are) the closest to the MWSN service customer in terms of their characteristics. In this step, a negotiation process between the service provider(s) and the service customer(s) takes place to reach a common SLA.

- The third step of the SLA contract lifecycle takes place when the service becomes operational (is started and is provided to the customer). During this step - which is called the SLA monitoring - the customer monitors and validates the service parameters offered by the MWSN provider.

- The last step when discussing about a SLA contract lifecycle is the SLA termination. In this step, the customer or the provider decide to terminate the agreement because the agreement expires or the agreement was violated by either the customer or the provider. In addition to the contract termination step there might exist yet another one that is called "enforce penalties for violation" [20].

\section{SLA parameters}

Different parameters can be defined when discussing about the SLA between the monitoring Wireless Sensor Networks (MWSNs) services providers and the MWSNs customers (see Table 1). When the choice of parameters for the SLA is lacking sufficient expertise then the SLA cannot be enforced automatically, and the service offered by the MWSN provider might behave in a wrong way. The term "sufficient expertise" might refer to the expertise of the engineers that design of the SLA of the MWSN service provider and/ or the service customers. This article attempts to support both the MWSN providers and the MWSN customers in selecting the appropriate 
Table 1. SLA parameters that measure the performance of a Monitoring Wireless Sensor Network (MWSN) service

\begin{tabular}{|l|l|l|}
\hline No & \multicolumn{1}{|c|}{ Name } & \multicolumn{1}{c|}{ Unit } \\
\hline 1. & Service availability & Time hour, percent \\
\hline 2. & (Maximum) down-time & Hours or percent \\
\hline 3. & Monitoring Wireless Sensor Network failure rate & Number \\
\hline 4. & Periods of operation & Time \\
\hline 5. & Latency times & ms \\
\hline 6. & Accessibility in case of problems & Yes/No \\
\hline 7. & Number and types of nodes & Number and type \\
\hline 8. & Energy consumed & Watt \\
\hline
\end{tabular}

parameters when designing the SLAs for MWSNs. In addition, in the monitoring step, based on the values of the SLA parameters, the participants can identify the causes that generate an erroneous behaviour of the MWSN.

Next, we propose some SLA parameters that are significantly related to the contract that characterizes the functionality of a MWSN both from the perspective of a MWSN provider and from the perspective of the MWSN customer.

In the case of a Monitoring Wireless Sensor Network (MWSN) service we can describe the SLA parameters that measure the service performance presented in Table 1. The parameters describe the infrastructure characteristics when discussing about a Monitoring Wireless Sensor Network.

Next, we discuss about each parameter of the MWSN that belongs to the SLA.

\subsection{Service availability}

A very important performance parameter when referring at a MWSN service is the service availability. In an environment that is prone to failures like a MWSN various incidents might occur like packet loss because of the malfunctioning of one or more nodes, communication delay, very volatile bandwidth and so on. During the malfunctioning of the MWSN service we can consider that the service is unavailable to the customer. This parameter is defined as the fraction of time the service is available to a customer [5]. Thus, the Service Availability A is defined usually by A = MTTF / (MTTF + MTTR), where MTTF represents the Mean Time To Failure, and MTTR defines the Mean Time To Repair. Usually the MTTR is much smaller than the MTTF. Opposed to the Service Availability is the Service Unavailability, given by the formula $\mathbf{U}=\mathbf{1}$ - $\mathbf{A}$ [4]

The Service Unavailability is the ratio of a timespan when the service is unavailable or offline. Service Levels and Guarantees a.k.a. SLA rules [14] related to service availability are values that can have high/low/median ranges. A SLA between the service provider and the service customer might state about the average service availability: "The average service availability must have a low value of $95 \%$, a high level of $99 \%$ and a median of $97 \%$ ".

The purpose of the definition of these values is to verify if the values surpass, are equal or are below the service level that is defined in the SLA at a certain point in time or in a certain validity time range. In the SLA contract, we have some generic rules in the form "if-then" [14] which describe the actions to be taken if some of the service availability thresholds are violated. For example, the following rule taken from [14] describes the consequential actions that are in place after violating an SLA service availability rule: "If the average service availability during one month is below $95 \%$ then the service provider is obliged to pay a penalty of $10 \%$ ".

\subsection{Maximum down-time}

The second performance parameter when discussing about MWSNs service is the service maximum down-time. A down-time can be planned or unplanned. Usually the planned downtime is denoted in the SLA contract as a particular maintenance time period when usual operations 
such as repairs, upgrades or other changes take place. In the case of the unplanned down-time, this parameter is defined to consider the possible situations when the service might be stopped due to the incorrect functioning of the MWSN either because of system failures (hardware failures) or because of the communication failures (network failures). These failures can be detected by using predictive monitoring. There is a need for MWSN monitoring tools that have the capability to predict and/or detect a malfunction in the MWSN. Another way to minimize the MWSN down-time is to have in place a mechanism of recovery from failure based a on certain type of routing (e.g. multipath routing [8], [1], [16]).

When specified in SLAs the maximum downtime is usually defined as a percentage value (per month or per year) that is computed as a sum of all the downtimes time spans and by dividing this sum by the total time of a reference time span (a month or a year). In the ideal case $0 \%$ maximum downtime specifies that the MWSN service was functional all the time.

A generic, about the MWSN maximum downtime, SLA rule in the form "if-then" can be the following: "If the maximum down-time for one month is above $0.1 \%$ then the service provider is obliged to pay a penalty of $10 \%$ ".

\subsection{MWSN failure rate}

MWSN failure rate is defined by the rate with which the MWSN stops functioning and is expressed by number of failures per unit of time. When discussing about the failure rate this parameter usually varies with the period which defines the life cycle of the network. For example, a MWSN that it is in its first month of service usually has a failure rate smaller than the failure rate of a MWSN that is in its third year of service. Failure rates are very important in defining the reliability of the MWSN [18]. To calculate the failure rate usually the MWSN is observed over a certain period and the time when the MWSN breaks down is recorded [18]. Because the MWSN failure rate (usually denoted by the Greek letter $\lambda$ (lambda)) is a statistical parameter, if there is a need to optimize the evaluation of the parameter $\lambda$, as much data as possible is needed. For example, if you want to compute the failure rate of a specific MWSN network, the result is better if you tested a MWSN over a greater period, like over a few months, rather than over a few days.
Example: Let us consider that we want to estimate the failure rate of a certain MWSN. The solution is to test a number of five identical MWSNs. We have the following data:

1. MWSN 1 failed after 500 hours.

2. MWSN 2 failed after 600 hours.

3. MWSN 3 failed after 700 hours.

4. MWSN 4 failed after 800 hours.

5. MWSN 5 functioned for 1000 hours.

By summing the 5 operation times we can see that we have 4 failures over 3600 functioning hours. When computing the failure rate, we can deduce that we have the MWSN failure rate, $\lambda=4 / 3600$ $=0.0011$ failures per hour.

Another way to compute the failure rate is by using the Mean Time Between Failures which is expressed by the value $\mathbf{M T B F}=\mathbf{1} / \boldsymbol{\lambda}$. In our example, the MTBF $=\mathbf{9 0 0}$ hours. This formula is used when there is a need to recommend to the MWSN administrators how often should a MWSN be inspected, maintained and replaced [18].

A generic, about the MWSN failure rate, SLA rule in the form "if-then" can be the following: "If the failure rate for one year is above 0.0011 failures per hour then the service provider is obliged to pay a penalty of 5\%".

\subsection{MWSN periods of operation}

MWSN periods of operation refers to the fact that the MWSN can operate during a given period during a day, during a month or even a year. The MWSN operates like described above because of various factors such as:

- the MWSN needs a human administrator to monitor the MWSN during a given schedule (for example during the week-end the human administrator doesn't work);

- the sensors need to be replaced after some time (for example every two months, and the replacement lasts for two hours);

- the application that monitors the MWSN needs to be updated after a certain period (for example every four months, operation that lasts for at most four hours);

- the customer negotiates in the SLA contract with the MWSN provider a specific 
operating schedule (for example daily between 8:00 and 20:00), because this period of operation is of interest for him.

A generic, about the MWSN period of operation, SLA rule in the form "if-then" can be the following: "If the MWSN period of operation is not between 8:00 and 20:00 daily then after the first two failures to have such values of the period of operation, the service provider is obliged to pay a penalty of $10 \%$ for every new violation".

\subsection{MWSN latency times}

MWSN latency times represents a temporal interval defined by the time it takes a bit of data to travel across the MWSN from one node called source to another node called destination or sink [19]. Minimizing latency is very important for data aggregation which is an essential application in MWSN [11], [22]. Usually the MWSN provider specifies in its MWSN latency times in terms of fractions of seconds (e.g. ms) or multiples of seconds (minutes). The customer is usually interested in the global MWSN latency, and the provider specifies this latency as a maximum and average latency [19].

A generic, about the MWSN latency times, SLA rule in the form "if-then" can be the following: "If the MWSN latency times is bigger than $100 \mathrm{~ms}$ then after two such violations the service provider is obliged to pay a penalty of $5 \%$ for every new violation".

\subsection{MWSN accessibility}

MWSN accessibility in case of problems represents a parameter that has two possible values: "Yes" or "No". During the negotiation phase of the SLA contract the MWSN provider agrees with the MWSN customer either to allow the customer to access the MWSN infrastructure to modify some parameters or not. Usually the customer does not have the expertise necessary to perform repairs, upgrades or other changes, thus the usual value for the parameter accessibility in case of problems is "No". In the case the value of this parameter is "Yes" then the customer can perform repairs, upgrades or other changes on the MWSN with or without (depending on how it was agreed on the SLA) the permission of the MWSN provider.

A generic, about the MWSN accessibility, SLA rule in the form "if-then" can be the following: "If the MWSN accessibility in case of problems parameter has the value No then the MWSN service customer cannot operate repairs, upgrades or other changes on the MWSN".

\subsection{MWSN number and type of nodes}

MWSN number and type of nodes parameters refers to how many monitoring nodes and their types were specified in the SLA contract. During the design phase of the SLA contract the customer formulates certain requirements in terms of necessary types of nodes and the number of functional nodes. The provider that signed the SLA contract agrees to offer these types of nodes and the corresponding number of functional nodes.

A generic, about the MWSN number of nodes, SLA rule in the form "if-then" can be the following: "If the MWSN number of nodes parameter has the value below the agreed value (e.g. 5 nodes) then the MWSN provider needs to solve this problem in 10 minutes otherwise it will pay a penalty of $10 \%$ for this violation. Similarly, a generic, about the MWSN types of nodes, SLA rule in the form "if-then" can be the following: "If the MWSN types of nodes parameter is smaller than the agreed ones (e.g. 5 different types of nodes) then the MWSN provider needs to solve this problem in 10 minutes otherwise it will pay a penalty of $10 \%$ for this violation".

\subsection{MWSN energy consumed}

MWSN energy consumed parameter refers at how much energy in terms of Watts the MWSN consumes. This parameter is very important from the point of view of the service provider, which is interested in minimizing the energy consumption of the MWSN. Several research papers have been published related to this aspect (e.g. [21], [17], [12]. Even if in the SLA contract it is not necessary to specify the energy consumption, research has been conducted in the area of energy consumed in MWSN in order to reduce costs.

In terms of costs a generic rule of the SLA in the form "if-then" can be the following: "If the monthly cost of a Monitoring Wireless Sensor Network is bigger than a specified sum per month (e.g. 100\$) then the MWSN customer can revoke the contract between him and the provider.

\section{Use case}

In this section, we want to demonstrate possible applications of MWSN in monitoring water 
quality, where there exists a SLA contract in place between the MWSN provider and the MWSN customer. The main purpose of such a MWSN is to automate the monitoring of drinking water quality. This section describes the SLA contract parameters based on previously published articles with the subject of Water Quality Monitoring Wireless Sensor Networks (WQMWSN).

In terms of availability one point of interest is the operation of establishing of high quality long-term WQMWSN. When creating a WQMWSN that has a high quality long term life there are several challenges that need to be surpassed such as [15]:

- there is a need for robust sensors because the operation of these sensors in a water environment can generate sensor malfunctioning; in the case of node(s) malfunctioning the node(s) that is(are) broken need(s) to be replaced;

- the process of sensor maintenance should be as less frequent as it is possible, and it should last as little time as it is possible;

- providing a power source (battery) that allows the WQMWSN provider to deploy the WQMWSN for as long time as possible; the life of the power source depends on various aspects such as the sensor(s) type and monitoring frequency.

If terms of down-time, usually the WQMWSN operates on batteries that are replaced when discharged. Additionally, a weekly calibration of the network must be done to get correct results [23]. Furthermore, there is a possibility that one or more nodes of the network doesn't function any more. In this case that down-time is minimal if the data transmitted by the WQMWSN is continuously monitored and actions are taken in consequence usually after a cycle time which is specified in the order of minutes: 20 minutes in [15], 30 minutes in [24], three minutes in [23]).

In terms of failure rate usually this parameter depends on the ability of the WQMWSN to function when one or more sensor node(s) stop(s) functioning. When there is a need to replace a sensor, this fact can be found by using a monitoring tool. The older the WQMWSN sensors are the bigger the failure rate is.

If we refer to the periods of operation usually we define these parameters as the periods of uninterrupted operation of the WQMWSN without the need to replace the WQMWSN battery (e.g. [24] 12 hours (the sensor node is active for 10 minutes, in [23] 40 days - the sensor node is active for 4 days).

If we consider latency of the WQMWSN we can say that this parameter can be expressed as the period between when the nodes of the WQMWSN go into sleep and when they become active plus the period of activity of the nodes $(60.039$ seconds sleep average in [15], 1800.1 seconds in [24], 1440 seconds in [23]).

In terms of accessibility of the WQMWSN the value of this parameter has usually the value No because the customer does not have the expertise necessary to perform repairs, upgrades or other changes and also because usually the monitoring process is performed in "a remote area with limited access" [24].

When discussing the WQMWSN number and type of nodes usually these parameters refer to the type of measurements the WQMWSN does. For example, in [15] there are six different types of nodes used, one of each (the number depends on the size of the WQMWSN):

I. Submersible Temperature Meter;

II. Water $\mathrm{pH}$ Meter;

III. Water Conductivity Meter;

IV. Dissolved Oxygen Sensor;

V. Water Turbidity Meter;

VI. Water Level Meter.

In [24] three types of sensor are used:

I. $\mathrm{pH}$ sensor that senses the acidity of the water,

II. temperature sensor and

III. turbidity sensor based on photo-transistor.

In [23] three types of measurements (thus sensors) are used:

I. fluctuations in $\mathrm{pH}$,

II. temperature,

III. dissolved oxygen.

The energy consumed parameter is defined in [15] as $96.2 \mathrm{~mW}$ for an active sensor, 0.054 $\mathrm{mW}$ for a sensor in sleep state, thus a medium power consumption value $0.116 \mathrm{~mW}$. In terms of percentage during the active period $53.6 \%$ it is consumed by a node when it is active, and $46.4 \%$ 
Table 2. SLA parameters and ways to optimize SLA design characteristics

\begin{tabular}{|c|c|}
\hline $\begin{array}{c}\text { SLA } \\
\text { parameter }\end{array}$ & How to optimize the design characteristic \\
\hline \multirow{2}{*}{ Availability } & Choose good quality, robust sensors \\
\hline & Use a powerful battery (with a long life) \\
\hline Down-time & Continuously monitor the WQMWSN \\
\hline Failure rate & Choose good quality, robust sensors \\
\hline $\begin{array}{l}\text { Periods of } \\
\text { operation }\end{array}$ & Choose a powerful battery (with a long life) \\
\hline Latency & $\begin{array}{l}\text { Choose the WQMWSN components and deploy them in such a way that } \\
\text { the sleep / wake up periods are in concordance with the necessities of the } \\
\text { WQMWSN customer and reduce the costs of the network }\end{array}$ \\
\hline Accessibility & $\begin{array}{l}\text { The provider offers either training if the customer needs to have access } \\
\text { to perform repairs, upgrades or other changes, or in the case access is not } \\
\text { required, the provider must have an optimal maintenance strategy for the } \\
\text { WQMWSN in place }\end{array}$ \\
\hline $\begin{array}{l}\text { Number } \\
\text { and types of } \\
\text { nodes }\end{array}$ & $\begin{array}{l}\text { The provider offers to the customer the type and number of sensors } \\
\text { required by the SLA contract }\end{array}$ \\
\hline $\begin{array}{c}\text { Energy } \\
\text { consumed }\end{array}$ & Choose low energy WQMWSN \\
\hline
\end{tabular}

during the period when it is in sleep state. In [24] when the node is active it consumes $0.3195 \mathrm{~W}$ and when it is in sleep state $0,2592 \mathrm{~W}$. This means that when in active state the node consumes $55.2 \%$ of the power and $44.8 \%$ of the power when in sleep mode.

The way to optimize the characteristics of a WQMWSN expressed in terms of SLA parameters are described in Table 2.

\section{Conclusions}

In this paper we discussed about defining parameters of a SLA contract that need to be considered during the SLA lifecycle. These parameters were also specified in terms "if-then rules" in order to have a clear understanding of how to build a SLA contract from the technical point of view. In addition, we presented also a use case of possible applications of MWSN in monitoring water quality. Our article is supposed to be a genuine help for both the MWSN providers and for the MWSN customers by explaining both the SLA lifecycle and the technical parameters that need to be specified on such a contract.

As future work we want to verify the SLA contract parameters described in this paper in real environments in order to further validate our paper.

\section{Acknowledgments}

The work supported in this paper was supported by Data4Water H2020 project and by Data4Water Nr.16/2016 (Premiere UEFISCDI).

\section{REFERENCES}

1. Al-Karaki, J. N. \& Kamal, A. E. (2004). Routing techniques in wireless sensor networks: a survey, IEEE wireless communications, 11(6), 6-28.

2. Casola, V., De Benedictis, A., Rak, M., Aversano, G. \& Villano, U. (2014). An SLA-based brokering platform to provide sensor networks as-a-service, International Journal of Business Process Integration and Management, 7(2), 114-127.

3. Casola, V., De Benedictis, A., Rak, M., Aversano, G. \& Villano, U. (2013, December). An SLA-based Approach to Manage Sensor Networks as-a-Service. In 2013 IEEE $5^{\text {th }}$ International Conference on Cloud Computing Technology and Science (CloudCom) (Vol. 1, pp. 191-197). IEEE.

4. Clouqueur, M. \& Grover, W. D. (2002). Availability analysis of span-restorable mesh networks, IEEE journal on selected areas in communications, 20(4), 810-821. 
5. Durvy, M., Diot, C., Taft, N. \& Thiranm, P. (2003). Network availability based service differentiation. In International Workshop on Quality of Service (pp. 305-325). Springer, Berlin, Heidelberg.

6. Gaillard, G., Barthel, D., Theoleyre, F. \& Valois, F. (2014, May). Service Level Agreements for Wireless Sensor Networks: A WSN operator's point of view. In Network Operations and Management Symposium (NOMS), 2014 IEEE (pp. 1-8). IEEE.

7. Gaillard, G., Barthel, D., Theoleyre, F. \& Valois, F. (2014). SLA Specification for IoT Operation-The WSN-SLA Framework (Doctoral dissertation, INRIA).

8. Ganesan, D., Ramesh, G., Shenker, S. \& Estrin, D. (2001). Highly-resilient, energyefficient multipath routing in wireless sensor networks, ACM SIGMOBILE Mobile Computing and Communications Review, 5(4), 11-25.

9. Iordache, G. V., Pop, F., Esposito, C., \& Castiglione, A. (2017). Selection-based Scheduling Algorithms under Service Level Agreement Constraints. In CSCS21: The 21 th International Conference on Control Systems and Computer Science. Bucharest, Romania.

10. Keller, A. \& Ludwig, H. (2003). The WSLA framework: Specifying and monitoring service level agreements for web services, Journal of Network and Systems Management, 11(1), 57-81.

11. Li, H., Wu, C., Hua, Q.-S. \& Lau, F. C. M. (2014). Latency-minimizing data aggregation in wireless sensor networks under physical interference model, $A d H o c$ Networks, 12, 52-68.

12. Manjeshwar, A. \& Dharma, P. A. (2001). TEEN: a routing protocol for enhanced efficiency in wireless sensor networks, Null, 30189a. IEEE.

13. Negru, C., Pop, F., Mocanu, M. \& Cristea, V. (2017, May). Storage Solution of SpatialTemporal Data for Water Monitoring Infrastructures Used in Smart Cities. In 2017 $21^{\text {st }}$ International Conference on Control Systems and Computer Science (CSCS) (pp. 617-621). IEEE.

14. Paschke, A. \& Schnappinger-Gerull, E. (2006). A Categorization Scheme for SLA Metrics, Service Oriented Electronic Commerce, 80(14), 25-40.
15. Regan, F., Lawlor, A., Flynn, O. B., Torres, J., Martinez-Catala, R., O'Mathuna, C. \& Wallace, J. (2009). A demonstration of wireless sensing for long term monitoring of water quality. In IEEE $34^{\text {th }}$ Conference on Local Computer Networks, LCN 2009 (pp. 819-825). IEEE.

16. Sohrabi, K., Gao, J., Ailawadhi, V. \& Pottie, G. J. (2000). Protocols for self-organization of a wireless sensor network, IEEE personal communications, 7(5), 16-27.

17. Van Dam, T. \& Langendoen, K. (2003). An adaptive energy-efficient MAC protocol for wireless sensor networks. In Proceedings of the $1^{\text {st }}$ International Conference on Embedded Networked Sensor Systems (pp. 171-180). ACM.

18. Weedmark, D., "How to Calculate Failure Rates", <http://sciencing.com/calculatefailure-rates-6403358.html $>$, last accessed 2017/10/03.

19. Wikipedia website, "Network delay page", $<$ https://en.wikipedia.org/wiki/Network delay $>$, last accessed 2017/10/03.

20. Wu, L. \& Buyya, R. (2012). Service level agreement (sla) in utility computing systems, IGI Global, 15.

21. Ye, W., Heidemann, J. \& Estrin, D. (2002). An energy-efficient MAC protocol for wireless sensor networks. In. Proceedings of the Twenty-First Annual Joint Conference of the IEEE Computer and Communications Societies, INFOCOM 2002 (Vol. 3, pp. 15671576). IEEE.

22. Yick, J., Biswanath, M. \& Ghosal, D. (2008). Wireless sensor network survey, Computer networks, 52(12), 2292-2330.

23. Zennaro, M., Athanasios, F., Dogan, G., Sun, T., Cao, Z., Huang, C., Bahader, M. \& Bagula A. (2009). On the design of a water quality wireless sensor network (wqwsn): An application to water quality monitoring in malawi. In International Conference on Parallel Processing Workshops ICPPW'09 (pp. 330-336). IEEE.

24. Zulhani, R. \& Mohd Rizal, A. (2009). Water quality monitoring system using zigbee based wireless sensor network, International Journal of Engineering \& Technology IJET, 9(10), 24-28. 